\title{
Iterative Solutions of Hybrid Integral Equations for Coexisting Open and Closed Surfaces
}

\author{
Özgür Ergül, Student Member, IEEE, and Levent Gürel, Fellow, IEEE
}

\begin{abstract}
We consider electromagnetics problems involving composite geometries with coexisting open and closed conductors. Hybrid integral equations are presented to improve the efficiency of the solutions, compared to the conventional electric-field integral equation. We investigate the convergence characteristics of iterative solutions of large composite problems with the multilevel fast multipole algorithm. Following a thorough study of how the convergence characteristics depends on the problem geometry, formulation, and iterative solvers, we provide concrete guidelines for efficient solutions.
\end{abstract}

Index Terms-Hybrid formulations, iterative methods, multilevel fast multipole algorithm, surface integral equations.

\section{INTRODUCTION}

$\mathbf{R}$ EAL-LIFE electromagnetics problems often involve both thin and thick conductors that need to be modeled with open and closed surfaces, respectively. Since the magnetic-field integral equation (MFIE) [1]-[4] is not applicable to open surfaces, conventional solutions employ the electric-field integral equation (EFIE) [5] even when only a small part of the geometry is an open surface. Unfortunately, EFIE usually gives ill-conditioned matrix equations that are difficult to solve with an iterative solver [6], especially when the problem includes some closed parts [7]. Consequently, as problem size increases, the solution of composite problems with coexisting open and closed surfaces becomes extremely difficult. To remedy this, we recently proposed a hybrid integral equation (HIE) [8]-[10], which uses the extended definition of the combined-field integral equation (CFIE) [2], [3]. This hybrid technique allows for more efficient solutions of composite problems without sacrificing accuracy.

HIE employs CFIE on the closed parts of the geometry, while EFIE is applied on the open parts, as usual. Mathematically, this corresponds to choosing different variables for the convex combination $^{1}$ of EFIE and MFIE depending on the row of the

Manuscript received August 31, 2008; revised December 14, 2008. Current version published June 03, 2009. This work was supported in part by the Scientific and Technical Research Council of Turkey (TUBITAK) under Research Grants 105E172 and 107E136, in part by the Turkish Academy of Sciences in the framework of the Young Scientist Award Program (LG/TUBA-GEBIP/ 2002-1-12), and in part by contracts from ASELSAN and SSM.

The authors are with the Computational Electromagnetics Research Center (BiLCEM) and the Department of Electrical and Electronics Engineering, Bilkent University, TR-06800 Bilkent, Ankara, Turkey (e-mail: ergul@ee.bilkent.edu.tr; lgurel@bilkent.edu.tr).

Color versions of one or more of the figures in this paper are available online at http://ieeexplore.iee.org.

Digital Object Identifier 10.1109/TAP.2009.2019921

${ }^{1} \mathrm{~A}$ convex combination is a linear combination, where all weights are nonnegative and they sum up to unity. matrix equation. Including of the boundary conditions on the magnetic field for the closed parts significantly improves the conditioning of the system. On the other hand, efficiency of the solution still depends on the geometry and the relative amounts of open and closed parts in the problem.

In this paper, we extensively investigate the iterative solution of composite problems involving open and closed conducting surfaces. Numerous examples of these problems are presented with their solutions and the results obtained. The rest of the paper is organized as follows. We revise the integral equations and introduce hybrid formulations in the next section, followed by the discretization of the integral equations. Iterative solutions of composite problems are investigated in Section IV, where we present the details of the iterations and convergence characteristics. Finally, we conclude with a discussion of efficient solution strategies for different classes of composite problems.

\section{INTEGRAL-EQUATION FORMULATIONS}

For conducting surfaces, the boundary condition for the tangential electric field can be used to derive EFIE as

$$
\begin{aligned}
\hat{\boldsymbol{t}} \cdot i k \int_{S} d \boldsymbol{r}^{\prime}\left[\boldsymbol{J}\left(\boldsymbol{r}^{\prime}\right)+\frac{1}{k^{2}} \nabla^{\prime} \cdot \boldsymbol{J}\left(\boldsymbol{r}^{\prime}\right) \nabla\right] & g\left(\boldsymbol{r}, \boldsymbol{r}^{\prime}\right) \\
& =-\hat{\boldsymbol{t}} \cdot \eta^{-1} \boldsymbol{E}^{\mathrm{inc}}(\boldsymbol{r})
\end{aligned}
$$

where we assume the $e^{-i \omega t}$ convention in phasor notation. In (1), the scattered field is expressed in terms of the induced (unknown) surface current $\boldsymbol{J}, \boldsymbol{r}$ is an observation point on the surface, $\hat{\boldsymbol{t}}$ is any tangential vector to the surface at the observation point, $\boldsymbol{E}^{\text {inc }}$ is the incident electric field, $\eta$ is the impedance of the host medium, $k=\omega \sqrt{\epsilon \mu}=2 \pi / \lambda$ is the wavenumber, and

$$
g\left(\boldsymbol{r}, \boldsymbol{r}^{\prime}\right)=\frac{e^{i k R}}{4 \pi R} \quad\left(R=\left|\boldsymbol{r}-\boldsymbol{r}^{\prime}\right|\right)
$$

denotes the homogeneous-space Green's function. An open surface can be thought of as the limit case of squeezing a closed surface into zero thickness so that the open surface is formed by merging the two opposite surfaces of an infinitely thin geometry. Then, the current distribution on the two sides of the thin structure becomes indistinguishable, and they are simply combined into a single equivalent current to be solved as the unknown of the problem [11]. For open surfaces, EFIE in (1) is still applicable since the implied boundary condition remains valid in this limit case. 
Similar to EFIE, MFIE is derived by using the boundary condition for the tangential magnetic field on the surface as

$$
-\boldsymbol{J}(\boldsymbol{r})+\hat{\boldsymbol{n}} \times \int_{S} d \boldsymbol{r}^{\prime} \boldsymbol{J}\left(\boldsymbol{r}^{\prime}\right) \times \nabla^{\prime} g\left(\boldsymbol{r}, \boldsymbol{r}^{\prime}\right)=-\hat{\boldsymbol{n}} \times \boldsymbol{H}^{\mathrm{inc}}(\boldsymbol{r})
$$

where the observation point approaches the closed surface $S$ from the outside, $\hat{\boldsymbol{n}}$ is the outwardly directed normal, and $\boldsymbol{H}^{\text {inc }}$ is the incident magnetic field. Although the current term $\boldsymbol{J}$ in (1) and (3) are the same, MFIE in (3) is not applicable to open surfaces. In addition to this, the MFIE formulation of a closed conductor becomes increasingly ill-conditioned as the geometry becomes thin and approaches an open surface.

The conventional CFIE is a convex combination of EFIE and MFIE [12], i.e.,

$$
\mathrm{CFIE}=\alpha \mathrm{EFIE}+(1-\alpha) \mathrm{MFIE}
$$

where $\alpha$ is a parameter between 0 and 1 . There are two main reasons why CFIE is the preferred integral equation for the solution of closed surfaces. First, it leads to better-conditioned matrix equations, compared to EFIE and MFIE [6], [7]; and second, it is free of the internal resonances experienced by EFIE and MFIE [2]. Since it contains MFIE however, CFIE in (4) is not applicable to open surfaces.

Extending the definition of CFIE, HIEs are obtained as

$$
\mathrm{HIE}=\alpha(\boldsymbol{r}) \mathrm{EFIE}+[1-\alpha(\boldsymbol{r})] \mathrm{MFIE}
$$

where this time the parameter $\alpha$ depends on the observation point. The use of this definition will be clear in the discretization of the equations, where we find a way to accelerate the iterative solution of composite problems.

\section{DISCRETIZATION OF THE INTEGRAL EQUATIONS}

Expanding the unknown surface current in a series of basis functions $\boldsymbol{b}_{n}$ as

$$
\boldsymbol{J}(\boldsymbol{r})=\sum_{n=1}^{N} a_{n} \boldsymbol{b}_{n}(\boldsymbol{r})
$$

the boundary conditions are projected onto the testing functions $\boldsymbol{t}_{m}$ to obtain $N \times N$ matrix equations as

$$
\sum_{n=1}^{N} Z_{m n}^{E, M} a_{n}=v_{m}^{E, M}, \quad m=1, \ldots, N .
$$

In (7), $a_{n}$ are the coefficients to be solved, and the matrix elements are derived by using (1) as

$$
\begin{aligned}
Z_{m n}^{E}=i k \int_{S_{m}} d \boldsymbol{r} t_{m}(\boldsymbol{r}) \cdot \int_{S_{n}} d \boldsymbol{r}^{\prime} g\left(\boldsymbol{r}, \boldsymbol{r}^{\prime}\right) \boldsymbol{b}_{n}\left(\boldsymbol{r}^{\prime}\right) \\
+\frac{i}{k} \int_{S_{m}} d \boldsymbol{r} t_{m}(\boldsymbol{r}) \cdot \int_{S_{n}} d \boldsymbol{r}^{\prime} \nabla^{\prime} \cdot \boldsymbol{b}_{n}\left(\boldsymbol{r}^{\prime}\right) \nabla g\left(\boldsymbol{r}, \boldsymbol{r}^{\prime}\right)
\end{aligned}
$$

for EFIE, and by using (3) as

$$
\begin{aligned}
Z_{m n}^{M}=-\int_{S_{m}} d \boldsymbol{r} \boldsymbol{t}_{m}(\boldsymbol{r}) \cdot \boldsymbol{b}_{n}(\boldsymbol{r}) & +\int_{S_{m}} d \boldsymbol{r t}_{m}(\boldsymbol{r}) \cdot \hat{\boldsymbol{n}} \\
& \times \int_{S_{n}} d \boldsymbol{r}^{\prime} \boldsymbol{b}_{n}\left(\boldsymbol{r}^{\prime}\right) \times \nabla^{\prime} g\left(\boldsymbol{r}, \boldsymbol{r}^{\prime}\right)
\end{aligned}
$$

for MFIE. Similarly, the elements of the excitation vector on the right-hand side (RHS) of (7) can be derived as

$$
v_{m}^{E}=-\eta^{-1} \int_{S_{m}} d \boldsymbol{r} \boldsymbol{t}_{m}(\boldsymbol{r}) \cdot \boldsymbol{E}^{\mathrm{inc}}(\boldsymbol{r})
$$

and

$$
v_{m}^{M}=-\int_{S_{m}} d \boldsymbol{r} t_{m}(\boldsymbol{r}) \cdot \hat{\boldsymbol{n}} \times \boldsymbol{H}^{\mathrm{inc}}(\boldsymbol{r})
$$

for EFIE and MFIE, respectively.

Using the definition in (4), the CFIE system is written as

$$
\begin{aligned}
Z_{m n}^{C} & =\alpha Z_{m n}^{E}+(1-\alpha) Z_{m n}^{M} \\
v_{m}^{C} & =\alpha v_{m}^{E}+(1-\alpha) v_{m}^{M}
\end{aligned}
$$

by forming the convex combinations of the matrix elements in (8) and (9) and the RHS vector elements in (10) and (11). Finally, for the hybrid formulations, we use a different combination parameter $\alpha_{m}$ for each testing function to arrive at

$$
\begin{aligned}
Z_{m n}^{H} & =\alpha_{m} Z_{m n}^{E}+\left(1-\alpha_{m}\right) Z_{m n}^{M} \\
v_{m}^{H} & =\alpha_{m} v_{m}^{E}+\left(1-\alpha_{m}\right) v_{m}^{M} .
\end{aligned}
$$

The matrix equation in (14)-(15) has a variable $\alpha_{m}$ depending on the index of the row $(m)$ in the impedance matrix, i.e., the index of the testing function. This provides the freedom to choose different linear combinations for different testing functions, even using pure EFIE $\left(\alpha_{m}=1\right)$ or pure MFIE $\left(\alpha_{m}=0\right)$ for some $m$.

The significance of choosing a flexible $\alpha_{m}$ becomes apparent in the solution of composite problems involving both open and closed surfaces. Even if the open parts are only a small portion of these problems, MFIE and CFIE are not applicable. Therefore, EFIE is the only choice despite its ill-conditioned nature, which is even more significant when applied on closed surfaces. Consequently, as the problem size gets larger and the number of unknowns $(N)$ increases, the solution of these problems becomes extremely difficult. Although the iterations are accelerated via efficient algorithms, such as the multilevel fast multipole algorithm (MLFMA) [12], [13], convergence is usually not reached within a reasonable number of iterations. To remedy this, it is possible with a hybrid formulation to use CFIE for the testing functions located on the closed parts of the geometry, while setting $\alpha_{m}=1$ to use EFIE for the open parts. We obtain better-conditioned systems by including the CFIE interactions in the solution of these problems. 
A hybrid formulation was employed in a previous study to avoid calculating both MFIE and EFIE interactions for all testing cases, thus reducing the computational cost of the matrix-filling [14]. Another study used the same idea to properly formulate electromagnetics problems with composite structures of thin and thick bodies that are completely closed [15]. There are many other studies presenting various hybrid formulations, where EFIE and MFIE are applied on different parts of the geometry and the two formulations are coupled via iterative updates of the equivalent sources [16]. In this paper, we use the extended definition with a variable $\alpha_{m}$ for the accurate and efficient iterative solutions of large problems with structures composed of both open and closed surfaces.

\section{Iterative SOlutions OF COMPOSITE PROBlems}

In this paper, composite problems are solved iteratively by using MLFMA to perform the matrix-vector multiplications efficiently. As detailed in [12], [13], MLFMA calculates the distant interactions between the basis and testing functions in a group-by-group manner consisting of three stages called aggregation, translation, and disaggregation. The MLFMA solution of HIEs, where $\alpha_{m}$ depends on the location of the testing function, is similar to the MLFMA solutions of EFIE, MFIE, and CFIE, and does not require any special procedures. This is because, in MLFMA, radiated and incoming fields do not depend on the type of the formulation and $\alpha_{m}$ appears only in the receiving patterns of the testing functions [12].

In all solutions, we employ Rao-Wilton-Glisson [17] functions, and geometries are triangulated with a mesh size of about $\lambda / 10$. Fig. 1 shows three different kinds of composite problems, where the relative amounts of open and closed surfaces are different. All objects are located in free space. The problems are also summarized in Table I. The first problem in Fig. 1(a) is a radiation problem involving a dipole antenna (open surface) of length $1 \mathrm{~m}$ located over a perfectly conducting rectangular box (closed surface) with the dimensions of $1 \mathrm{~m} \times 5 \mathrm{~m} \times$ $5 \mathrm{~m}$. This structure is an example of problems with almost completely closed geometries, except for very minor open parts. The problem is solved at $300 \mathrm{MHz}$, and the discretization of the object leads to 24,186 unknowns on the box and only 19 unknowns on the antenna. We simulated the feed of the antenna by a delta-gap source located at the center of the strip, as shown in Fig. 1(a). A local electric field with amplitude $1 / d$ is defined inside the infinitely narrow opening between the triangles so that

$$
\begin{aligned}
v_{m}^{H} & =\alpha_{m} v_{m}^{E}+\left(1-\alpha_{m}\right) v_{m}^{M}=v_{m}^{E} \\
& =-\lim _{d \rightarrow 0} \eta^{-1} \int_{S_{m}} d \boldsymbol{r} \boldsymbol{t}_{m}(\boldsymbol{r}) \cdot \boldsymbol{E}^{\mathrm{inc}}(\boldsymbol{r}) \\
& = \pm \eta^{-1} l_{e} \delta[m, e]
\end{aligned}
$$

where

$$
\delta[m, e]=\left\{\begin{array}{ll}
1 & m=e \\
0 & m \neq e
\end{array}\right\} .
$$

In (16), $l_{e}$ is the length of the edge $e$, on which the delta-gap source is defined.

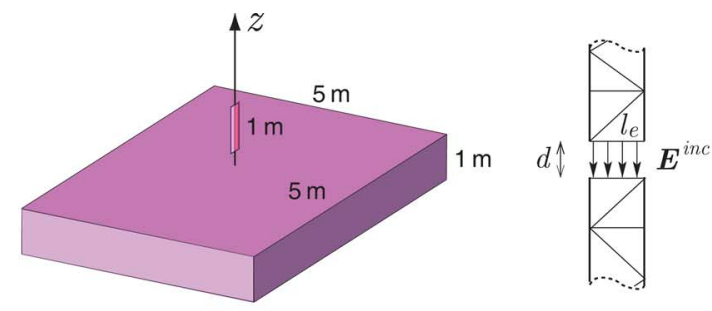

(a)

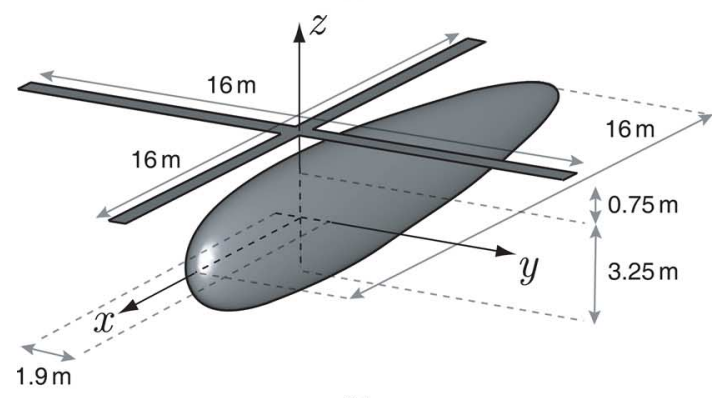

(b)

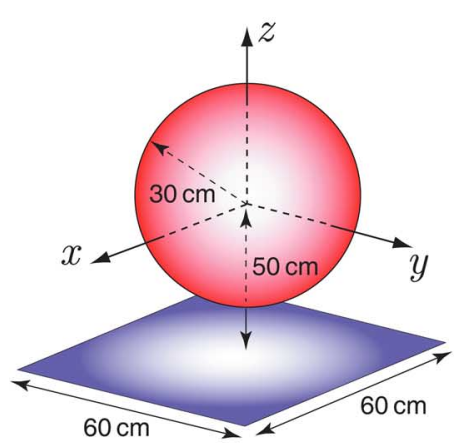

(c)

Fig. 1. Various composite problems (a) with almost completely closed geometries, except for very minor open parts, (b) involving more closed parts than open parts, and (c) with comparable amounts of open and closed parts.

TABLE I

CoMposite PROBLEMS

\begin{tabular}{|c|c|c|c|}
\hline & & \multicolumn{2}{|c|}{ Number of Unknowns } \\
\hline Problem & Frequency & Closed Parts & Open Parts \\
\hline Problem 1: Fig.1(a) & $300 \mathrm{MHz}$ & 24,186 & 19 \\
\hline Problem 2: Fig.1(b) & $500 \mathrm{MHz}$ & 115,023 & 16,608 \\
\hline Problem 3: Fig.1(c) & $1 \mathrm{GHz}$ & 3768 & 1301 \\
\hline Problem 3: Fig.1(c) & $6 \mathrm{GHz}$ & 132,336 & 49,200 \\
\hline
\end{tabular}

Fig. 1(b) shows an example of problems involving mostly closed parts but with some open parts. A cross-shaped open surface is placed over a closed ellipsoid surface with the dimensions shown in the figure. The object is investigated at $500 \mathrm{MHz}$ and illuminated by a plane wave propagating in the $-x$ direction with the electric field polarized in the $y$ direction. Discretization of the object leads to 115,023 unknowns on the ellipsoid (closed surface) and 16,608 unknowns on the cross-shaped part (open surface).

Finally, as an example of problems with comparable amounts of open and closed parts, Fig. 1(c) shows a sphere with radius $30 \mathrm{~cm}$ placed over a $60 \mathrm{~cm} \times 60 \mathrm{~cm}$ square patch. The structure is illuminated by the same plane wave as in the second problem. The problem is solved at two frequencies; at $1 \mathrm{GHz}$, 


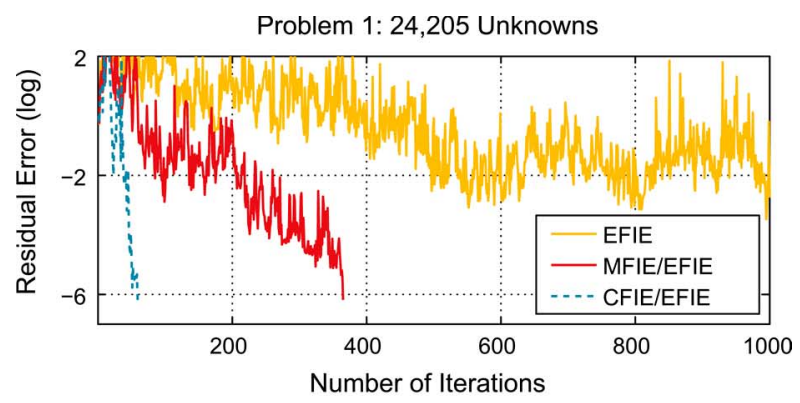

(a)

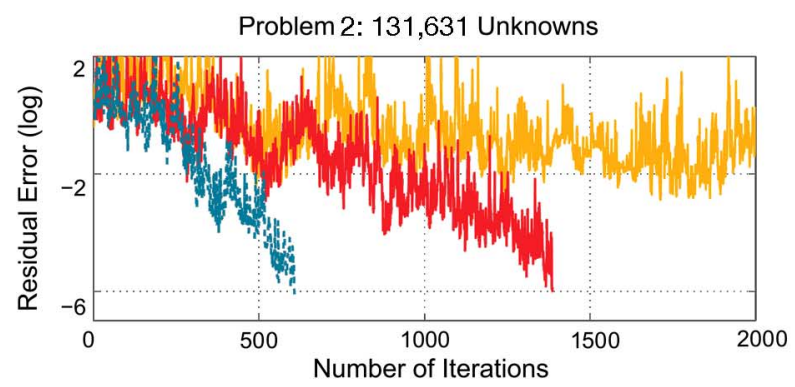

(b)

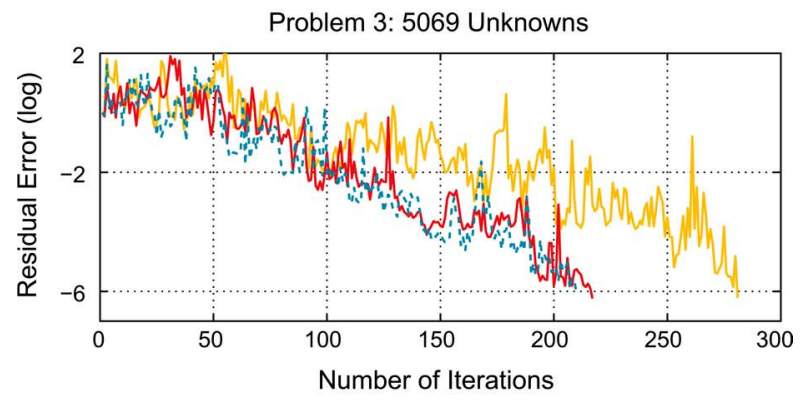

(c)

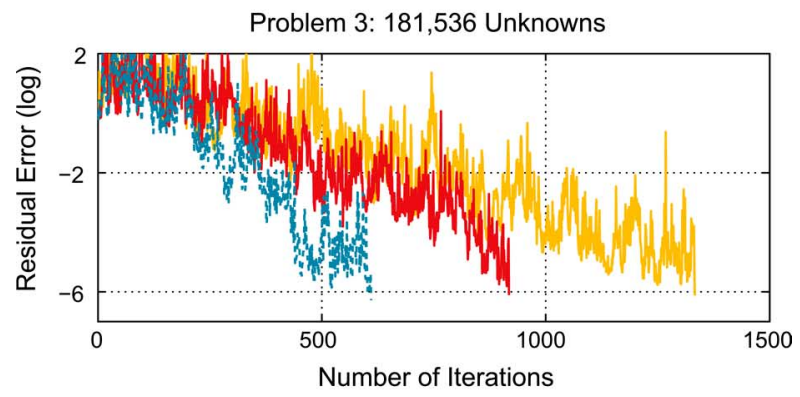

(d)

Fig. 2. Convergence characteristics of the problems in Fig. 1 for different formulations, i.e., EFIE, MFIE/EFIE, and CFIE/EFIE, solved with CGS.

the number of unknowns is 3768 on the sphere and 1301 on the patch, whereas at $6 \mathrm{GHz}$, there are 132,336 unknowns on the sphere and 49,200 on the patch.

\section{A. Formulation}

In Fig. 2, convergence characteristics are depicted for the conjugate gradient squared (CGS) algorithm and for different formulations applied to the problems defined above. In all examples, iterative convergence is the slowest for the conventional EFIE. Especially for the first two problems, EFIE does not reach the $10^{-6}$ residual error within a reasonable number of iterations.

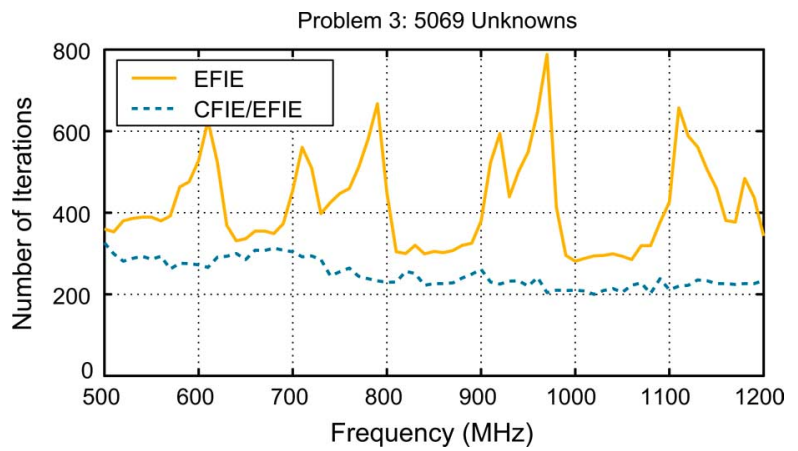

Fig. 3. Iteration counts to reach $10^{-6}$ residual error for the problem in Fig. 1(c) in the frequency range from $500 \mathrm{MHz}$ to $1200 \mathrm{MHz}$.

As shown in Fig. 2(a) and (b), the hybrid formulation dramatically improves the convergence of these two problems, when CFIE with $\alpha_{m}=0.2$ is applied to the closed parts. (The choice of $\alpha_{m}$ will be clarified in the next subsections.) On the other hand, when MFIE is applied to the closed parts, leading to a hybrid MFIE/EFIE formulation, improvement is not sufficient.

For the third problem containing comparable amounts of open and closed surfaces, there is improvement with the hybrid CFIE/EFIE formulation, but it is less than those in the previous two problems. For this problem, EFIE converges better than the first and second problems due to the larger portion of open surface. The CFIE/EFIE formulation is still much better than EFIE for the larger problem in Fig. 2(d). However, the acceleration provided by CFIE/EFIE is not very significant for the smaller problem in Fig. 2(c). In fact, the iterations in Fig. 2(c) are at the frequency where EFIE shows quite good performance. In Fig. 3, a frequency sweep from $500 \mathrm{MHz}$ to $1200 \mathrm{MHz}$ is demonstrated for the same problem. EFIE suffers from internal resonances, and the number of iterations peaks at various frequencies. On the other hand, the hybrid CFIE/EFIE formulation is free of the internal resonances so that the number of iterations is steady and consistently in the same low range.

\section{B. Iterative Methods}

We have been using the following iterative methods [18] for the solution of electromagnetics problems formulated with integral equations [19]:

- BiCG: Biconjugate gradient;

- BiCGStab: Biconjugate gradient stabilized;

- CGS: Conjugate gradient squared;

- GMRES: Generalized minimal residual;

- LSQR: Least-square QR;

- TFQMR: Transpose-free quasi-minimal residual.

As an example, we present the solution of Problem 1 with various iterative methods. Fig. 4 demonstrates the convergence characteristics of the solutions for different formulations, where we observe that the hybrid CFIE/EFIE formulation consistently leads to the fastest convergence. Table II lists the number of iterations for the same problem to reach $10^{-6}$ residual error. When the EFIE formulation is applied to the entire problem, CGS, LSQR, and TFQMR converge within 2000 iterations, while BiCG, BiCGStab, and GMRES (with a restart parameter of 200 iterations) do not. With CFIE/EFIE, the number of iterations drops for all cases, but GMRES performs the best in 

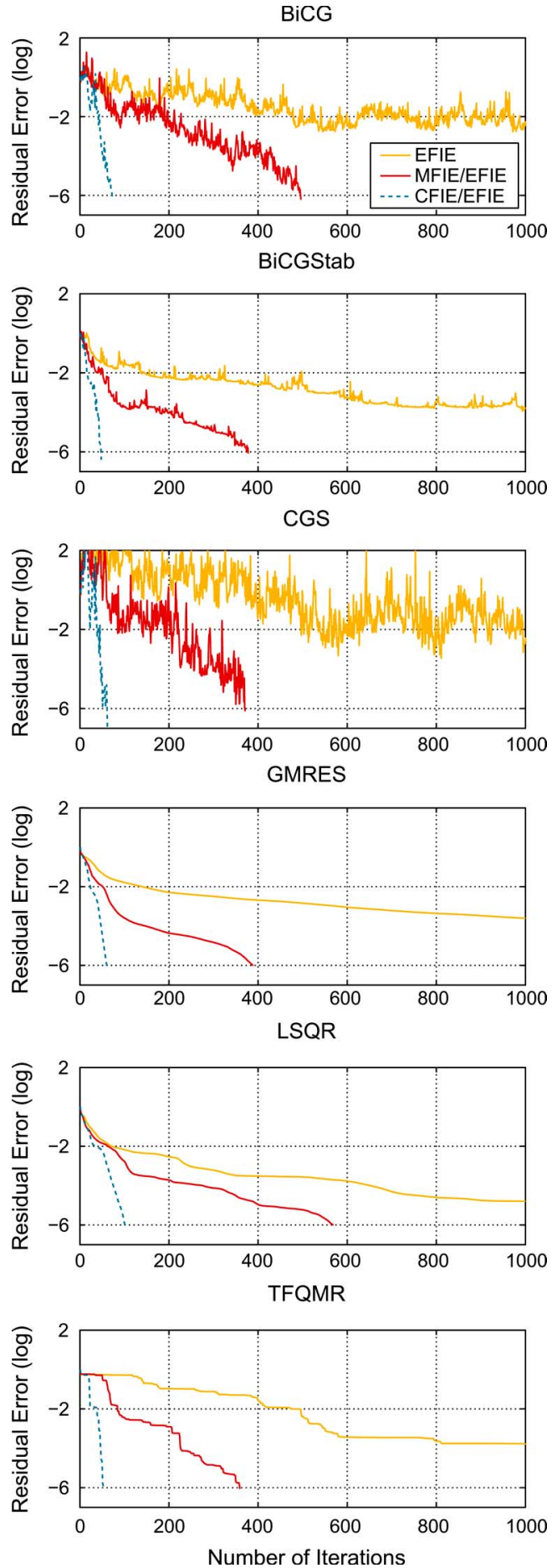

Fig. 4. Convergence characteristics of the problem in Fig. 1(a) for various iterative methods and different formulations, i.e., EFIE, MFIE/EFIE, and CFIE/ EFIE.

terms of the number of matrix-vector multiplications. This is because GMRES requires only one matrix-vector multiplication per iteration while the others require two. On the other hand, GMRES requires more memory than the other iterative methods in Table II; hence BiCGStab might be preferred as problem size grows and it becomes critical to reduce memory.
TABLE II

NUMBER OF ITERATIONS FOR PROBLEM 1 TO REACH $10^{-6}$ RESIDUAL ERROR

\begin{tabular}{|c|c|c|c|}
\hline Method & EFIE & MFIE/EFIE & CFIE/EFIE \\
\hline BiCG & $>2000$ & 495 & 72 \\
\hline BiCGStab & $>2000$ & 377 & 47 \\
\hline CGS & 1488 & 372 & 63 \\
\hline GMRES & $>2000$ & 388 & 60 \\
\hline LSQR & 1307 & 568 & 101 \\
\hline TFQMR & 1407 & 358 & 52 \\
\hline
\end{tabular}

\section{Preconditioning}

In the iterative solution of the matrix equation

$$
\bar{Z} \cdot \boldsymbol{a}=\boldsymbol{v}
$$

the system can be transformed into a new system as

$$
\bar{M}^{-1} \cdot \bar{Z} \cdot \boldsymbol{a}=\bar{M}^{-1} \cdot \boldsymbol{v}
$$

or as

$$
\bar{Z} \cdot \bar{M}^{-1} \cdot \boldsymbol{b}=\boldsymbol{v}
$$

where

$$
\boldsymbol{a}=\bar{M}^{-1} \cdot \boldsymbol{b} .
$$

In (19) and (20), the matrix $\bar{M}$ approximates $\bar{Z}$ so that the transformed system is likely to be better-conditioned than the original system. In MLFMA implementations, all the near-field interactions are available for the construction of a strong preconditioner. For example, with the direct factorization of the sparse near-field matrix of all near-field interactions, we obtain the near-field preconditioner (NFP) [7]. However, both the factorization performed once before the iterations and the solution of the preconditioner system applied in each iteration are expensive in terms of processing time; thus the preconditioner becomes a bottleneck as problem size grows. To remedy this, a block diagonal preconditioner (BDP) that is much more efficient can be obtained by extracting only the self interactions of the lowest-level clusters [13]. Both the factorization and application of the BDP are $\mathcal{O}(N)$; this is appropriate for MLFMA since the complexity of MLFMA is $\mathcal{O}(N \log N)$ and the complexity of the preconditioner should not be higher than that. However, BDP is not always successful in reducing the number of iterations.

Convergence characteristics for the preconditioned systems are depicted in Fig. 5. For the three problems, EFIE and hybrid formulations are solved with CGS preconditioned by BDPs. The EFIE solutions with NFP are also presented in the same figures for comparison. To easily check the effect of preconditioning on different problems and formulations, Table III lists the number of iterations to reach $10^{-6}$ residual error. Looking at Fig. 5 and Table III, we note the following observations.

- For the first problem with a completely closed geometry except for a very minor open part, the convergence of the 


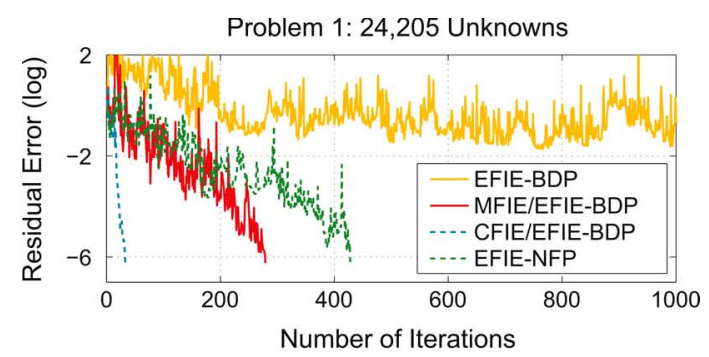

(a)

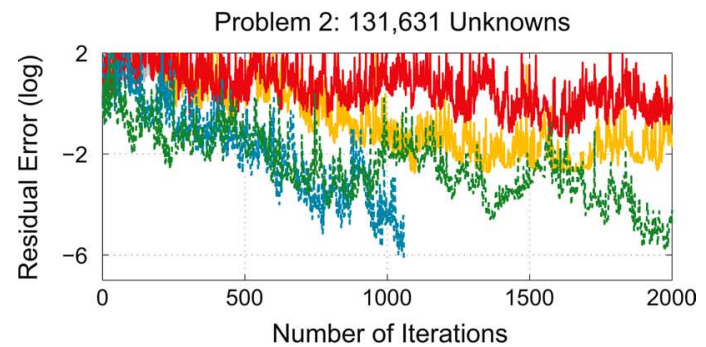

(b)

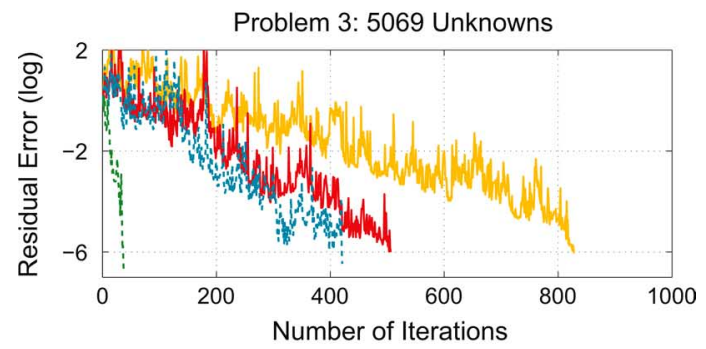

(c)

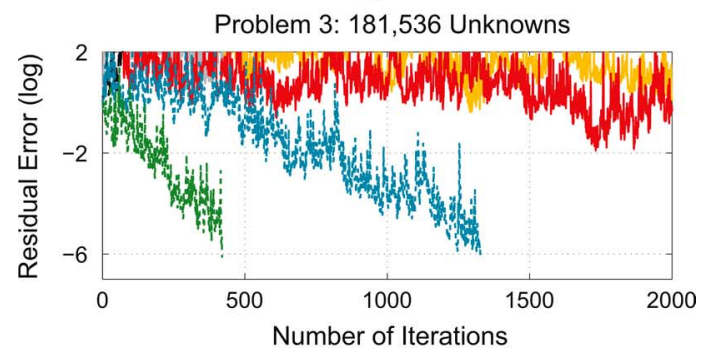

(d)

Fig. 5. Convergence characteristics of the problems in Fig. 1 for the preconditioned CGS and different formulations, i.e., EFIE, MFIE/EFIE, and CFIE/EFIE.

hybrid formulations is significantly improved by the efficient BDP. However, for EFIE, BDP does not work and the convergence is worse than the no-preconditioner (NP) case. The strong NFP improves the convergence of EFIE, although the number of iterations is still high, compared to the hybrid formulations.

- For the second problem, where there are more closed than open parts, BDP does not work even with the hybrid formulations. This can be explained with the worsening of the condition of the system since more open parts need to be formulated with EFIE, compared to the first problem. Nevertheless, the hybrid CFIE/EFIE formulation is preferable since its NP case is still better than EFIE with a strong NFP.

- For the third problem, where the open and closed parts are comparable, the effect of EFIE becomes significant. As in the second problem, BDP does not work even for the hybrid
TABLE III

NUMBER OF CGS ITERATIONS TO REACH $10^{-6}$ RESIDUAL ERROR

\begin{tabular}{|l|c|c|c|c|}
\hline Problem/Unknowns & PC & EFIE & MFIE/EFIE & CFIE/EFIE \\
\hline Problem 1 / 24,205 & NP & 1488 & 372 & 63 \\
\hline Problem 1 / 24,205 & BDP & $>2000$ & 280 & 33 \\
\hline Problem 1 / 24,205 & NFP & 429 & - & - \\
\hline \hline Problem 2 / 131,631 & NP & $>2000$ & 1387 & 607 \\
\hline Problem 2 / 131,631 & BDP & $>2000$ & $>2000$ & 1058 \\
\hline Problem 2 / 131,631 & NFP & $>2000$ & - & - \\
\hline \hline Problem 3 / 5069 & NP & 280 & 218 & 219 \\
\hline Problem 3 / 5069 & BDP & 828 & 507 & 421 \\
\hline Problem 3 / 5069 & NFP & 37 & - & - \\
\hline \hline Problem 3 / 181,536 & NP & 1334 & 919 & 611 \\
\hline Problem 3 / 181,536 & BDP & $>2000$ & $>2000$ & 1327 \\
\hline Problem 3 / 181,536 & NFP & 420 & - & - \\
\hline
\end{tabular}

formulations. In addition to this, EFIE with NFP performs better than the hybrid formulations with NP. Consequently, NFP provides a trade-off between memory and number of iterations. There is an alternative way (not shown in this paper) using the hybrid CFIE/EFIE formulation with NFP and leading to fewer iterations. However, due to the non-symmetric impedance matrix, this would be more expensive in terms of memory than the EFIE case.

\section{CFIE Variable}

Finally, we focus on the variable $\alpha_{m}$ employed in the convex combination of EFIE and MFIE in the CFIE part of the hybrid formulations. We demonstrate two different cases in Fig. 6, where the number of CGS iterations are depicted with respect to the variable $\alpha_{m}$ applied on the closed parts of the problems. The four curves in each figure represent the residual errors from $10^{-3}$ to $10^{-6}$. Comparing Fig. 6(a) and (b), we observe that the dependence on the $\alpha_{m}$ is directly based on the type of geometry. In Problem 1, where only a small portion of the geometry is open, there is an optimal $\alpha_{m}$ of about $0.2-0.4$ for the best convergence. In both directions to MFIE/EFIE $\left(\alpha_{m}=0\right)$ and to EFIE $\left(\alpha_{m}=1\right)$, the number of iterations increases. On the other hand, in the case of Problem 3, the open part is relatively larger, and it is difficult to set an optimal $\alpha_{m}$ for $\alpha_{m} \neq 1$. We conclude that choosing $\alpha_{m}$ around 0.2 does not guarantee the exact optimal convergence for all cases, but it provides a number of iterations close to the optimal case.

\section{RCS RESULTS}

To check the accuracy of the hybrid formulations, radar cross section (RCS) results are presented for the problems in Fig. 1(a) and (c). The normalized RCS is defined as

$$
\frac{\operatorname{RCS}(\theta, \phi)}{\lambda^{2}}=\frac{1}{\pi} \lim _{r \rightarrow 0}\left\{(k r)^{2} \frac{\left|\boldsymbol{E}^{\text {sca }}(r, \theta, \phi)\right|^{2}}{\left|\boldsymbol{E}^{\text {inc }}(r, \theta, \phi)\right|^{2}}\right\}
$$

for any direction $(\theta, \phi)$, where $\boldsymbol{E}^{\text {sca }}$ and $\boldsymbol{E}^{\text {inc }}$ represent the scattered and incident electric fields. In Fig. 7, the normalized RCS values $(\mathrm{dB})$ are plotted on the $z-x$ plane with respect to the $\theta$ angle defined as increasing from the positive $z$ axis to the positive $x$ axis. The highest RCS values in Fig. 7(b) and (c) appear at $270^{\circ}$, which corresponds to the forward-scattering direction according to the excitations described in Section IV. For 


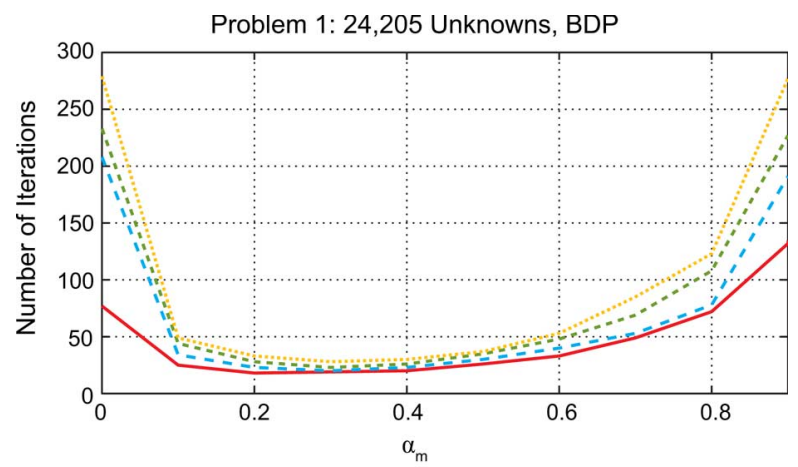

(a)

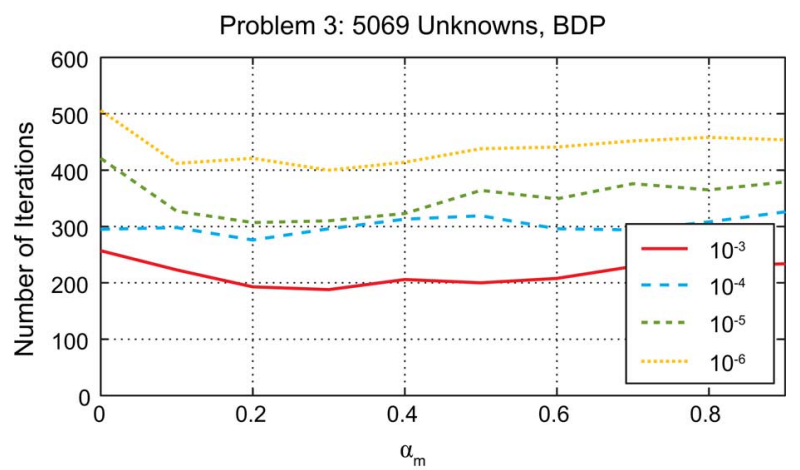

(b)

Fig. 6. Iteration counts with respect to the variable $\alpha_{m}$, which is applied to the closed parts of the problems.

both radiation and scattering problems, we observe that the hybrid CFIE/EFIE formulation provides accurate results that agree well with the reference EFIE solutions.

\section{CONCLUDING REMARKS}

In this study, we investigate the effect of the HIE formulations specifically for the iterative solutions of composite problems involving open and closed conducting surfaces. The results indicate that the iterative solutions are significantly improved with the hybrid formulations, compared to the conventional EFIE. Based on an extensive set of results, some of which are presented in this paper, we offer the following guidelines for the iterative solutions of the composite problems.

1) For problems with closed geometries except for very minor parts [such as Problem 1 in Fig. 1(a)], use the hybrid CFIE/ EFIE formulation with $\alpha_{m}=0.2$ and employ BDP to accelerate the solution. GMRES and BiCGStab are good candidates for the iterative solver.

2) For problems with less open parts and more closed parts [such as Problem 2 in Fig. 1(b)], use the hybrid CFIE/ EFIE formulation with $\alpha_{m}=0.2$. Avoid using simple preconditioners such as BDP that is likely to slow down the convergence.

3) For problems with comparable open and closed parts [such as Problem 3 in Fig. 1(c)], use the hybrid formulation with $\alpha_{m}=0.2$. Again, avoid using simple preconditioners such as BDP. On the other hand, if the memory is available, EFIE with a strong preconditioner (e.g., NFP) is still a

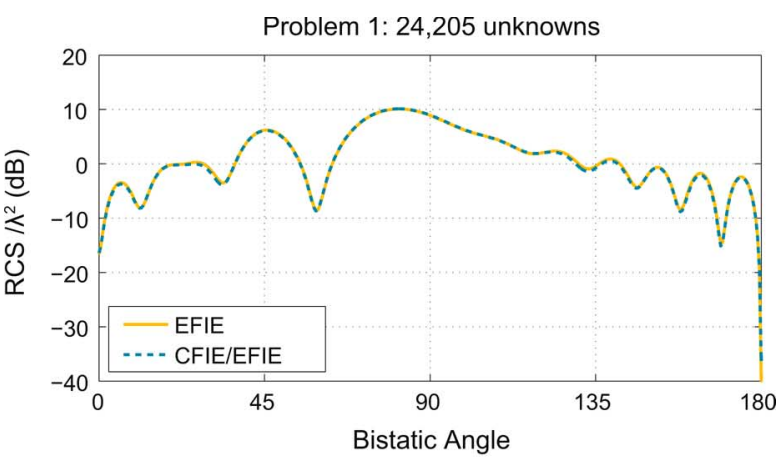

(a)

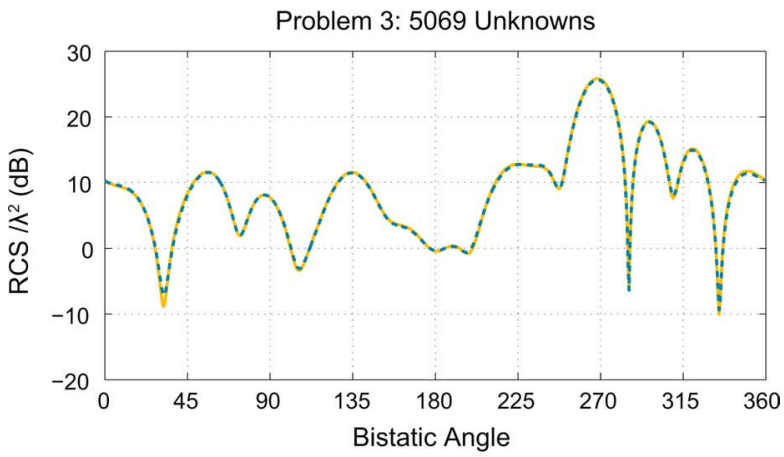

(b)

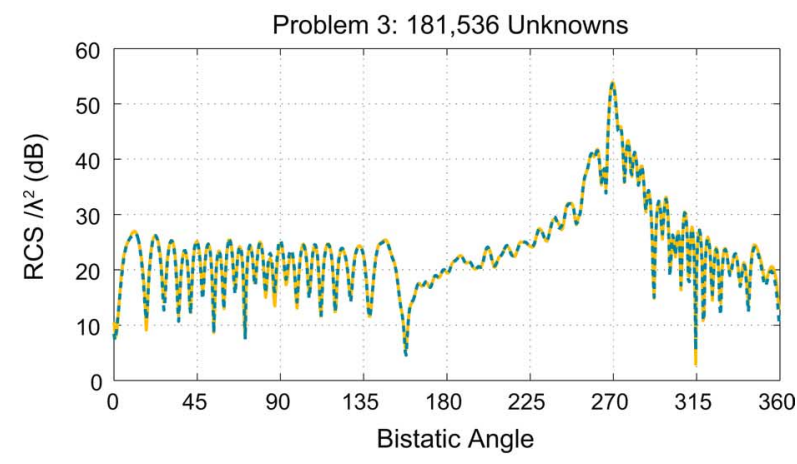

(c)

Fig. 7. Bistatic RCS values on the $z-x$ plane. The angle $\theta$ is defined to start from the $+z$ axis and increase toward the $+x$ axis.

good choice and may perform better than the hybrid formulations without a preconditioner. Similar to problems involving only open surfaces [19], LSQR may perform better than the other iterative methods for this type of composite problems.

\section{REFERENCES}

[1] A. J. Poggio and E. K. Miller, "Integral equation solutions of three-dimensional scattering problems," in Computer Techniques for Electromagnetics, R. Mittra, Ed. Oxford, U.K.: Pergamon Press, 1973, ch. 4.

[2] J. R. Mautz and R. F. Harrington, "H-field, E-field, and combined field solutions for conducting bodies of revolution," $A E \ddot{U}$, vol. 32, no. 4, pp. 157-164, Apr. 1978.

[3] N. Morita, N. Kumagai, and J. R. Mautz, Integral Equation Methods for Electromagnetics. Boston, MA: Artech House, 1990.

[4] Y. Zhang, T. J. Cui, W. C. Chew, and J.-S. Zhao, "Magnetic field integral equation at very low frequencies," IEEE Trans. Antennas Propag., vol. 51, no. 8, pp. 1864-1871, Aug. 2003. 
[5] A. W. Glisson and D. R. Wilton, "Simple and efficient numerical methods for problems of electromagnetic radiation and scattering from surfaces," IEEE Trans. Antennas Propag., vol. 28, no. 5, pp. 593-603, Oct. 1980.

[6] D. R. Wilton and J. E. Wheeler, III, "Comparison of convergence rates of the conjugate gradient method applied to various integral equation formulations," Progr. Electromagn. Res., pp. 131-158, 1991.

[7] Ö. Ergül and L. Gürel, "Comparisons of FMM implementations employing different formulations and iterative solvers," in Proc. IEEE Antennas Propag. Soc. Int. Symp., 2003, vol. 1, pp. 19-22.

[8] Ö. Ergül and L. Gürel, "Hybrid CFIE-EFIE solution of composite geometries with coexisting open and closed surfaces," in Proc. IEEE Antennas Propag. Soc. Int. Symp., 2005, vol. 4B, pp. 289-292.

[9] L. Gürel and Ö. Ergül, "Iterative solution of composite problems with the combined-field integral equation," in Proc. Eur. Microw. Conf., 2006, pp. 239-240.

[10] L. Gürel and Ö. Ergül, "Extending the applicability of the combinedfield integral equation to geometries containing open surfaces," IEEE Antennas Wireless Propag. Lett., vol. 5, pp. 515-516, 2006.

[11] A. F. Peterson, S. L. Ray, and R. Mittra, Computational Methods for Electromagnetics. New York: IEEE Press, 1998.

[12] W. C. Chew, J.-M. Jin, E. Michielssen, and J. Song, Fast and Efficient Algorithms in Computational Electromagnetics. Boston, MA: Artech House, 2001.

[13] J. Song, C.-C. Lu, and W. C. Chew, "Multilevel fast multipole algorithm for electromagnetic scattering by large complex objects," IEEE Trans. Antennas Propag., vol. 45, no. 10, pp. 1488-1493, Oct. 1997.

[14] A. Helaly and H. M. Fahmy, "Combined-field integral equation," Electron. Lett., vol. 29, no. 19, pp. 1678-1679, Sep. 1993.

[15] W. V. T. Rusch and R. J. Pogorzelski, "A mixed-field solution for scattering from composite bodies," IEEE Trans. Antennas Propag., vol. 34, no. 7, pp. 955-958, Jul. 1986.

[16] R. E. Hodges and T. Rahmat-Samii, "An iterative current-based hybrid method for complex structures," IEEE Trans. Antennas Propag., vol. 45, no. 2, pp. 265-276, Feb. 1997.

[17] S. M. Rao, D. R. Wilton, and A. W. Glisson, "Electromagnetic scattering by surfaces of arbitrary shape," IEEE Trans. Antennas Propag., vol. 30, no. 3, pp. 409-418, May 1982.

[18] S. Balay, K. Buschelman, V. Eijkhout, W. D. Gropp, D. Kaushik, M. G. Knepley, L. C. McInnes, B. F. Smith, and H. Zhang, PETSc Users Manual, Argonne National Laboratory, Argonne, IL, 2004.

[19] Ö. Ergül and L. Gürel, "Efficient solution of the electric-field integral equation using the iterative LSQR algorithm," IEEE Antennas Wireless Propag. Lett., vol. 7, pp. 36-39, 2008.

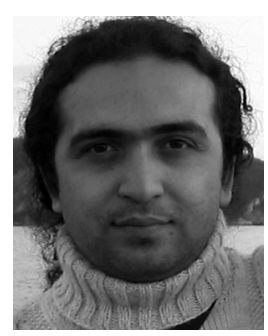

Özgür Ergül (S'98) received B.Sc. and M.S degrees in electrical and electronics engineering from Bilkent University, Ankara, Turkey, in 2001 and 2003, respectively. He is currently pursuing the Ph.D. degree at Bilkent University.

Since 2001, he has served as a Teaching and Research Assistant in the Department of Electrical and Electronics Engineering at Bilkent University. He has been affiliated with the Computational Electromagnetics Group at Bilkent University from 2000 to 2005 and with the Computational Electromagnetics Research Center (BiLCEM) since 2005. His research interests include fast and accurate algorithms for the solution of electromagnetics problems involving large and complicated structures, integral equations, parallel programming, and iterative techniques.

Mr. Ergül is a recipient of the 2007 IEEE Antennas and Propagation Society Graduate Fellowship and the 2007 Leopold B. Felsen Award for Excellence in Electrodynamics. He is the Secretary of Commission E (Electromagnetic Noise and Interference) of URSI Turkey National Committee. His academic endeavors are supported by the Scientific and Technical Research Council of Turkey (TUBITAK) through a Ph.D. scholarship.

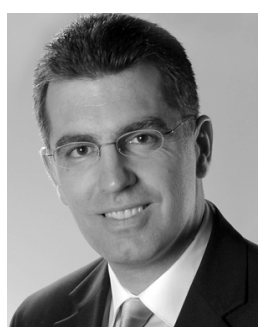

Levent Gürel (S'87-M'92-SM'97-F'09) received the B.Sc. degree from the Middle East Technical University (METU), Ankara, Turkey, in 1986, and the M.S. and Ph.D. degrees from the University of Illinois at Urbana-Champaign (UIUC), in 1988 and 1991, respectively, all in electrical engineering.

He joined the Thomas J. Watson Research Center of the International Business Machines Corporation, Yorktown Heights, New York, in 1991, where he worked as a Research Staff Member on the electromagnetic compatibility (EMC) problems related to electronic packaging, on the use of microwave processes in the manufacturing and testing of electronic circuits, and on the development of fast solvers for interconnect modeling. Since 1994, he has been a faculty member in the Department of Electrical and Electronics Engineering, Bilkent University, Ankara, where he is currently a Professor. He was a Visiting Associate Professor at the Center for Computational Electromagnetics (CCEM), UIUC for one semester in 1997. He returned to the UIUC as a Visiting Professor in 2003-2005, and as an Adjunct Professor after 2005. He founded the Computational Electromagnetics Research Center (BiLCEM) at Bilkent University in 2005 , where he is serving as the Director. His research interests include the development of fast algorithms for computational electromagnetics (CEM) and the application thereof to scattering and radiation problems involving large and complicated scatterers, antennas and radars, frequency-selective surfaces, high-speed electronic circuits, optical and imaging systems, nanostructures, and metamaterials. He is also interested in the theoretical and computational aspects of electromagnetic compatibility and interference analyses. Ground penetrating radars and other subsurface scattering applications are also among his research interests. Since 2006, his research group has been breaking several world records by solving extremely large integral-equation problems, most recently the largest involving as many as 205 million unknowns.

Prof. Gürel's many accomplishments include two prestigious awards from the Turkish Academy of Sciences (TUBA) in 2002 and the Scientific and Technical Research Council of Turkey (TUBITAK) in 2003. He served as the Chairman of the AP/MTT/ED/EMC Chapter of the IEEE Turkey Section in 2000-2003. He founded the IEEE EMC Chapter in Turkey in 2000. He served as the Cochairman of the 2003 IEEE International Symposium on Electromagnetic Compatibility. $\mathrm{He}$ is the organizer and General Chair of the CEM'07 ve CEM'09 Computational Electromagnetics International Workshops held in 2007 and 2009. He is a member of the USNC of the International Union of Radio Science (URSI) and the Chairman of Commission E (Electromagnetic Noise and Interference) of URSI Turkey National Committee. He served as a member of the General Assembly of the European Microwave Association (EuMA) during 2006-2008. $\mathrm{He}$ is currently serving as an associate editor for the IEEE ANTENNAS AND WIRELESS PROPAGATION LETTERS, Radio Science, Journal of Electromagnetic Waves and Applications (JEMWA), and Progress in Electromagnetics Research (PIER). 